\title{
Erratum zu: Lebensweg zu und Motive bei der Entscheidung, in der Kindertagespflege tätig zu sein - Explorative empirische Befunde
}

\author{
Juliane Noack Napoles • Helza Ricarte Lanz
}

(C) Die Autor(en) 2015. Dieser Artikel ist auf Springerlink.com mit Open Access verfügbar.

Im ursprünglichen Beitrag wurden die Angaben zur Autorin Helza Ricarte Lanz falsch wiedergegeben. Der korrekte Name und Anschrift lauten:

Helza Ricarte Lanz

Institut für Erziehungswissenschaft und empirische Bildungs- und Sozialforschung Alanus Hochschule für Kunst und Gesellschaft

hl@drlanz.org

Wir bedauern diesen Fehler vielmals.

Open Access Dieser Artikel unterliegt den Bedingungen der Creative Commons Attribution License. Dadurch sind die Nutzung, Verteilung und Reproduktion erlaubt, sofern der/die Originalautor/en und die Quelle angegeben sind.

Die Online-Version des Originalartikels ist unter doi:10.1007/s40955-015-0009-x zu finden

Dr. H. Ricarte Lanz ( $\square)$

Institut für Erziehungswissenschaft und empirische Bildungs- und Sozialforschung, Alanus Hochschule für Kunst und Gesellschaft,

Alfter, Deutschland

E-Mail: hl@drlanz.org

Dr. J. Noack Napoles

Institut für Bildungsphilosophie, Anthropologie und Pädagogik der Lebensspanne, Universität zu Köln,

Köln, Deutschland

E-Mail: juliane-noack@hotmail.com 